\title{
A REALIDADE AUMENTADA NA APRESENTAÇÃO DE PRODUTOS CARTOGRÁFICOS
}

\section{The Augmented Reality in the Presentation of Cartographic Products}

\author{
Wendson de Oliveira Souza ${ }^{1}$ \\ Giovana Mira de Espindola ${ }^{1,2}$ \\ Alessandro Rhadamek Alves Pereira ${ }^{1}$ \\ Lucilene Antunes Correia Marques de Sá ${ }^{3}$ \\ 1 Universidade Federal do Piauí - UFPI. Centro de Tecnologia, Departamento de Transportes. Teresina, \\ Piauí, Brasil. E-mail: wendsonsouza@ufpi.edu.br; alessandro.rhadamek@ufpi.edu.br. \\ 2 Universidade Federal do Piauí - UFPI. Programa de Pós-Graduação em Desenvolvimento e Meio \\ Ambiente (PRODEMA/TROPEN). Teresina, Piauí, Brasil. E mail: giovanamira@ufpi.edu.br. \\ ${ }^{3}$ Universidade Federal de Pernambuco - UFPE. Centro de Tecnologia e Geociências, Departamento de \\ Engenharia Cartográfica. Programa de Pós-Graduação em Ciências Geodésicas e Tecnologias da \\ Geoinformação. Recife, Pernambuco, Brasil. E-mail: lacms@ufpe.br.
}

\section{Resumo:}

A Realidade Aumentada (RA) é uma tecnologia com novo paradigma de interface com os usuários que vem crescendo em importância na apresentação de produtos cartográficos. A visualização de elementos naturais é incorporada à representação estática terrestre em um ambiente digital interativo. Essa transformação provoca mudanças na forma como os usuários percebem os produtos cartográficos que antes eram apresentados em meios analógicos. O uso da Realidade Aumentada potencializa a apresentação de produtos cartográficos tridimensionais dinâmicos, e permite ampliar o aspecto cognitivo de usuários não especialistas em contato com documentos cartográficos. A tecnologia remonta a década de 1960, e nos dias atuais tem se expandido em diversas funcionalidades em vários setores. $\mathrm{O}$ artigo discute a incorporação da Realidade Aumentada na construção do modelo 3D do campus da Universidade Federal de Pernambuco. Tal modelo foi utilizado em experimento aplicado a usuários não especialistas, tendo-se como eixos de avaliação: aspecto territorial, interpretação de elementos, e aspecto comunicativo. Os resultados do experimento mostram que o processo de ensino-aprendizagem na área da cartografia é um campo fértil para atuação da RA, tornando-se um ponto de partida para a elaboração de uma base cartográfica aumentada.

Palavras-chave: Visualização Cartográfica; Interatividade, Modelagem 3D.

\begin{abstract}
:
Augmented reality (AR) is a technology with new user interface paradigm that is growing in importance in the presentation of cartographic products. The display of natural elements is incorporated into the static terrestrial representation in an interactive digital environment. This
\end{abstract}


transformation causes changes in the way users perceive cartographic products that were presented in analog media. The use of augmented reality enhances the presentation of dynamic and three-dimensional cartographic products, and allows the enhancement of cognitive aspect of non-expert users in contact with cartographic documents. The technology dates back to the 1960s, and today has grown into several features in various sectors. The article discusses the incorporation of augmented reality in building the 3D model of the Federal University of Pernambuco campus. This model was used in an experiment applied to non-expert users, having as evaluation axes: territorial aspect, elements interpretation, and communicative aspect. The results of the experiment show that the teaching-learning process in the area of cartographic is a suitable ground for the performance of RA, becoming a starting point for the development of an augmented cartographic base map.

Keywords: Cartographic Visualization; Interactivity, 3D Modeling.

\section{Introdução}

O presente artigo, por meio de uma revisão da literatura, apresenta inicialmente uma visão geral dos fundamentos da Realidade Aumentada (RA), focando posteriormente na aplicação da RA no processo de ensino-aprendizagem na área da cartografia. Desta forma, busca-se discutir a integração da RA às técnicas cartográficas consolidadas, visando a construção de uma abordagem educacional iterativa.

Sabe-se, que ao longo dos anos, a cartografia tem se beneficiado do desenvolvimento de tecnologias que permitem a representação e visualização de informações tridimensionais do espaço físico estático de forma mais realística e dinâmica. Tais tecnologias permitem explorar os elementos da superfície terrestre como eles são e em diferentes perspectivas, e os exemplos mais comuns destas tecnologias são os Globos Virtuais, a Realidade Virtual e a Realidade Aumentada (Stanek e Friedmannova, 2010; Meneguette, 2012). Estas últimas são atualmente baseadas em serviços de internet (geo-services), e cada uma delas apresenta um nível específico de desafio para a cartografia, principalmente na área da visualização cartográfica.

$\mathrm{O}$ uso e as potencialidades dos globos virtuais e da realidade virtual na representação e percepção da realidade geográfica estão bem documentados na literatura recente, principalmente nas áreas do conhecimento que lidam com dados espaciais (Bailey e Chen, 2011; Bodenhamer, 2014; Harwood et al., 2015; Shekhar et al., 2015). No entanto, estudos que buscam discutir as potencialidades da Realidade Aumentada (RA) na cartografia, inserindo o uso desta tecnologia no âmbito da visualização cartográfica, são mais escassos. Isto porque, conceitualmente, a RA é dentre as tecnologias a mais distante conceitualmente da cartografia e a menos explorada (Shelton e Hedley, 2002; Stanek e Friedmannova, 2010).

Historicamente, o processo de assimilação de novas tecnologias na cartografia tem exigido diferentes entendimentos sobre as abordagens cartográficas em ambientes digitais, ou seja, sobre como representar dados espaciais em tais ambientes. A maneira mais simples e mais aceita num primeiro momento sempre foi a transferência dos antigos conceitos e técnicas ao novo ambiente virtual, como por exemplo, a apresentação de um mapa em tela, reproduzindo o que se faz em papel, sem se explorar as possibilidades de apresentar visões a partir de diferentes pontos de vista. Esta maneira é útil em algumas aplicações, mas não explora o uso e as potencialidades de 
novos ambientes digitais. Neste sentido, discussões acerca das possibilidades de aplicação da Realidade Aumentada no desenvolvimento de produtos cartográficos interativos é importante, e visa identificar as contribuições resultantes da união destas duas áreas do conhecimento.

Este artigo tem por objetivo discutir a utilização de Realidade Aumentada na construção de um modelo tridimensional correspondente ao campus da Universidade Federal de Pernambuco (UFPE) em Recife - PE, visando investigar a incorporação desse mecanismo à base cartográfica digital da área de estudo. Com base neste modelo, discute-se ainda por meio de um experimento com usuários não especialistas os benefícios do uso da RA no processo de ensino-aprendizagem na área da cartografia.

\section{Visualização cartográfica: conceito de interatividade}

A visualização cartográfica é o ramo da cartografia derivada da visualização científica, que tem por objetivo fornecer ferramentas e visualizações que visam facilitar o raciocínio espacial e a resolução de problemas, com ênfase não no armazenamento do conhecimento, mas sim na sua construção, tendo como base tecnologias computacionais que permitam a criação de resultados visuais interativos gerados exclusivamente por mídias digitais. Na visualização cartográfica, os mapas são apresentados como ferramentas de análise, que podem ser utilizadas tanto para transmitir as características dos fenômenos geográficos, como para sintetizar soluções ou mostrar resultados. Tal área tem sido bastante explorada na cartografia com trabalhos clássicos de MacEachren e colegas (DiBiase et al., 1992; Hearnshaw e Unwin, 1994; MacEachren e Kraak, 1997; MacEachren, 2004; MacEachren e Taylor, 2013).

A partir de Taylor (1991) e Taylor (1994) a visualização passou a figurar como disciplina da cartografia, tendo como principais aspectos a cognição e a análise, a comunicação, a apresentação e a formalização, sendo este último aspecto destinado à incorporação de técnicas computacionais e multimídia. A partir deste autor, MacEachren e Kraak (1997) conceituam a visualização como complemento da comunicação. A visualização é definida como a indução do pensamento visual por meio da descoberta de padrões de dados não conhecidos, enquanto que a comunicação é entendida como a transferência da informação. Qualquer leitura de um mapa inclui tanto a visualização quanto a comunicação, mas o uso do mapa pode diferir consideravelmente dependendo de qual dessas atividades se prioriza, ou seja, a distinção passa não pelos tipos de mapas, mas sim pelos tipos de usuários e pelo nível de interação homem-mapa (Meneguette, 2012).

Neste contexto, surge o termo interatividade, que tem, desde a década de 1970, se tornado cada vez mais importante nas estratégias de nível privado. Para Delgado et al. (2001) a interatividade é a possibilidade de transformar o usuário em emissor e receptor da mensagem. Neste trabalho, parte-se do entendimento de Silva e Gomes (2013) em que interatividade é um termo diretamente relacionado ao uso de tecnologias computacionais, sendo o ambiente computacional o lugar da intermediação entre o usuário e a máquina. 


\section{Realidade Aumentada: definição e aplicações}

A representação em terceira dimensão pode ser enriquecida por aspectos visuais (cor, saturação, luminosidade), por objetos em cena (árvore, poste de iluminação, placa de sinalização), e por movimentação sobre o modelo (andar, sobrevoar). A visualização tridimensional de um modelo, além de propor uma interface mais amigável e atraente ao usuário, mostra ser útil na execução de projetos, pois permite uma análise qualitativa direta e mais intuitiva e realista que os mapas convencionais, possibilitando até mesmo que usuários não especialistas possam interagir com as informações representadas (Fosse, 2004).

A modernização da cartografia em 3D proporciona praticidade na condução e exposição de informações geográficas. A Realidade Aumentada combina ambiente real com elementos virtuais. A utilização deste sistema na cartografia permite que camadas de informações de cunho analógico sejam exibidas de forma virtual, isto é, na representação de mapas 3D interativos. Em outras palavras, a RA é definida por um sistema que complementa o mundo físico com objetos virtuais, não restrito ao ambiente de computador. Com o advento de equipamentos móveis (tabletes, smartphones e óculos inteligentes) seu campo de aplicação é cada vez maior e se estende a várias áreas da ciência e da sociedade. No entanto, a RA difere dos sistemas de multiprojeção imersivos de realidade virtual do tipo caverna digital (Cruz-Neira et al., 1993, Zuffo et al., 2001), pois na RA existe um maior nível de iteratividade baseado na manipulação em tempo real dos modelos 3D.

As bases fundamentais e tecnológicas da RA remontam aos primórdios da pesquisa sobre computação gráfica. A era da computação gráfica teve início com o advento dos computadores equipados com recursos gráficos de simulação, animação, e visualização de dados numéricos (Azuma, 1997; Henderson e Feiner, 2007; Henderson e Feiner, 2011). No ano de 1968 foi apresentado por Ivan Sutherland um capacete HMD (Head Mounted Display) considerado como um dos trabalhos relevantes em visualização tridimensional, sendo informatizado por meio de um sistema óptico-sensor capaz de visualizar objetos em 3D e determinar a posição e a orientação do observador (Sutherland, 1968). As contribuições deste trabalho tiveram aceitação nas industriais aeroespaciais e automobilísticas americana, o que permitiu à General Motors desenvolver um dos primeiros softwares de CAD (Computer Aided Design).

Ao longo dos anos, outras inovações foram desenvolvidas, mas só a partir da década de 1990 a Realidade Aumentada adquire uma presença maior no mundo científico, beneficiada pelos avanços da computação, que através de métodos, algoritmos e do desenvolvimento de hardware possibilitaram a evolução de sistemas informáticos acessíveis e capazes de renderizar e apresentar conteúdos virtuais na forma de gráficos e em tempo real.

A RA difere da realidade virtual na medida em que utiliza elementos virtuais para complementar as informações que percebemos do mundo real, isto é, aumenta a realidade em vez de substituí-la inteiramente em um ambiente virtual, e assim torna-se possível expandir a percepção e interação, e aperfeiçoa a análise e tomada de decisão do usuário sobre eventos do mundo real (Azuma, 1997; Green et al., 2008; Van Krevelen e Poelman, 2010). Os objetos virtuais exibem informações que o usuário não pode detectar diretamente com seus próprios sentidos. A informação transmitida pelos objetos virtuais auxilia o usuário a executar suas tarefas no mundo real, tais como análises e tomadas de decisão. Azuma (1997) ressalta que as principais características da RA resultam da combinação de objetos reais e virtuais em ambiente real, ou seja, da interação em tempo real e registro 3D preciso de objetos virtuais e reais. 
A ação da RA não se limita ao ambiente fixo de computadores (desktop, notebook e laptop). Sua atuação se estende de forma crescente em dispositivos móveis (tabletes e smartphones), principalmente pelas opções encontradas em aparelhos com acesso à internet (wifi e 3G/4G), velocidade de processamento, sistema de navegação global por satélite (GNSS) e câmera. Há exemplo de aplicação móvel de RA via projeção de edifício 3D em espaço urbano, com possibilidade de navegação e interação em tempo real (Portalés et al., 2010).

A mobilidade potencializa as aplicações e interações da RA, propiciando aos usuários oportunidades de explorar novos ambientes (Pinto e Centeno, 2012). A experiência de aproximar as pessoas a elementos virtuais, postos em seu ambiente real, desperta o interesse de empresas como Google e Microsoft para o desenvolvimento de óculos inteligentes. Há óculos de funcionamento autônomo ou conectado a dispositivos portáteis com conexão à internet para geração de objetos virtuais instantâneos.

O Google Glass permite a projeção de elementos virtuais em uma das lentes no campo de visualização do observador. A tela exibe alternativas de informações da previsão do tempo, rotas de trajetória, mapas e outras opções. Por enquanto, o invento da Google foi retirado do mercado por problemas técnicos. Como alternativa promissora desponta o Microsoft HoloLens que é capaz de criar hologramas no campo de visão do usuário. O alcance do dispositivo projeta-se num raio de poucos metros no ambiente real onde é utilizado. As imagens holográficas 3D podem ser compartilhadas ao mesmo tempo por várias pessoas com a tecnologia, proporcionando experiências imersivas e interativas mútuas.

A RA apresenta outras funcionalidades de desenvolvimento e aplicações, e os recentes avanços ocorridos na interface de computadores associados com as tecnologias de comunicação fizeram expandir o uso e a atuação desta tecnologia a níveis superiores, o que proporcionou amplificar sua ação para além de jogos eletrônicos, publicidade, propaganda e entretenimento. Nos dias atuais, o desenvolvimento e as aplicações em sistema de Realidade Aumentada vêm ganhando espaço e destaque, e proporcionando melhorias em diversas áreas: setor automobilístico por meio do para-brisa do carro com informações do veículo, possibilitando maior segurança ao condutor, como acontece, por exemplo, no Jaguar XF (head-up display); procedimentos cirúrgicos, com localização precisa de vasos sanguíneos, auxiliando no tratamento de varizes (Miyake et al., 2006); mercado imobiliário, na avaliação da pós-ocupação de imóveis, permitindo evidenciar reformas ao ambiente construído, e outras.

No âmbito da educação, o processo de ensino e aprendizagem tem se beneficiado ao longo dos anos de recursos tecnológicos na construção do conhecimento. Aulas tradicionais começam muitas vezes a se tornar desinteressantes, quando comparadas às aulas desenvolvidas em meios tecnológicos. Ou seja, aulas mais envolventes e interativas, na medida em que a componente tecnológica e a prática pedagógica atuam de maneira integrada na condução de conteúdo, e na interação entre professor e aluno.

Assim, o uso da Realidade Aumentada pode-se fazer presente em distintas aplicações educacionais. Há atuações na educação básica como, por exemplo, no ensino da matemática com experimentos de geometria espacial, em ciências com demonstrações do sistema solar e simulação do aparelho digestivo, e ensino superior para a demonstração de conceitos de engenharia mecânica (Basogain et al., 2007; Cadavieco et al., 2012).

De modo geral, o processo de criação de um ambiente em Realidade Aumentada exige hardware de captura de informações, software para geração de elementos em tempo real e hardware para representar estes elementos no ambiente onde são inseridos (Kirner et al., 2004; Cardoso et al., 2007). Com isto é possível sobrepor elementos virtuais a algo real. Para tanto, emprega-se o rastreamento por marcador impresso (símbolo ou código) na utilização da técnica de visão computacional, Figura 1a. $\mathrm{O}$ uso de marcadores no rastreamento possibilita a inserção exata no 
mundo real pela geração de objetos virtuais a eles associados, além de permitir movimentos de rotação e translação.

A formação do objeto virtual se inicia quando o programa computacional processa a imagem capturada do marcador por uma câmera, que identifica a sua orientação e o seu posicionamento. O programa em RA disponibiliza um sistema baseado em algoritmos que faz a tradução da simbologia (código) representada no marcador, e o transforma em uma projeção virtual com base neste posicionamento. Essa informação possibilita a perspectiva correta da imagem 3D a ser projetada. Para que a imagem se torne visível, antes ela precisa ser criada em programas de modelagens 3D, em seguida, armazenada e vinculada ao marcador em uso no programa de RA. Com isso, a imagem capturada do marcador delimita o plano sobre o qual a ação da RA desenvolve o objeto 3D, combinando a cena atual com a imagem computadorizada em perspectiva real. A RA pode ser construída em tela de televisor, de monitor, de projetor ou de dispositivo portáteis, Figura $1 b$.

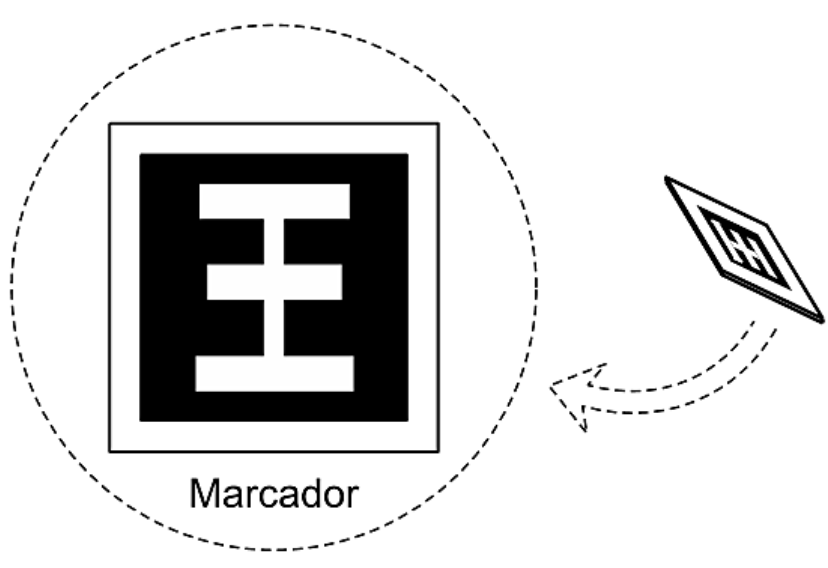

(a)

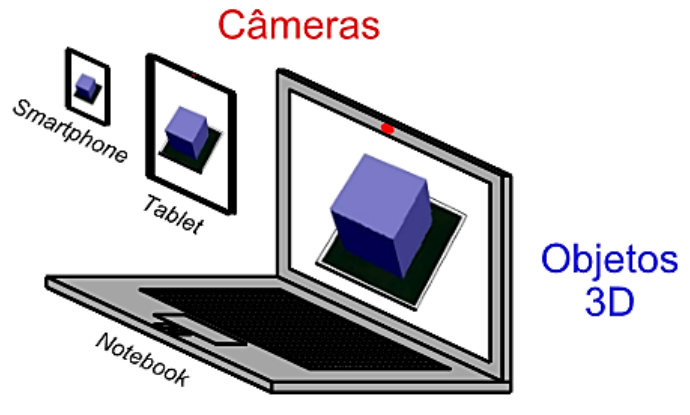

Hardware e Software

(b)

Figura 1: Sistemas de RA constituído por: (a) marcador; e (b) hardware e software.

\section{Caracterização da área de estudo}

O interior do campus Joaquim Ignácio de Almeida Amazonas, da Universidade Federal de Pernambuco, foi adotado como área de estudo para a criação da modelagem tridimensional. $\mathrm{O}$ campus encontra-se localizado no município de Recife, Pernambuco, sendo banhado pelo Riacho Cavouco (afluente do Rio Capibaribe) e cortado pela Rodovia Federal BR-101.

O campus universitário da UFPE foi fundado em 1948, e sua localização no bairro da Várzea (antigo Engenho do Meio) foi influenciada por existir uma avenida projetada para o local e pelas condições climáticas e topográficas do terreno, Figura 2. A cidade universitária possui aproximadamente 140 ha, e reúne mais de quarenta prédios, entre eles a Reitoria, nove Centros Acadêmicos, oito Órgãos Suplementares, Centro de Convenções, Concha Acústica, Clube Universitário, Creche, Casa do Estudante (masculina e feminina) e o Restaurante Universitário. 


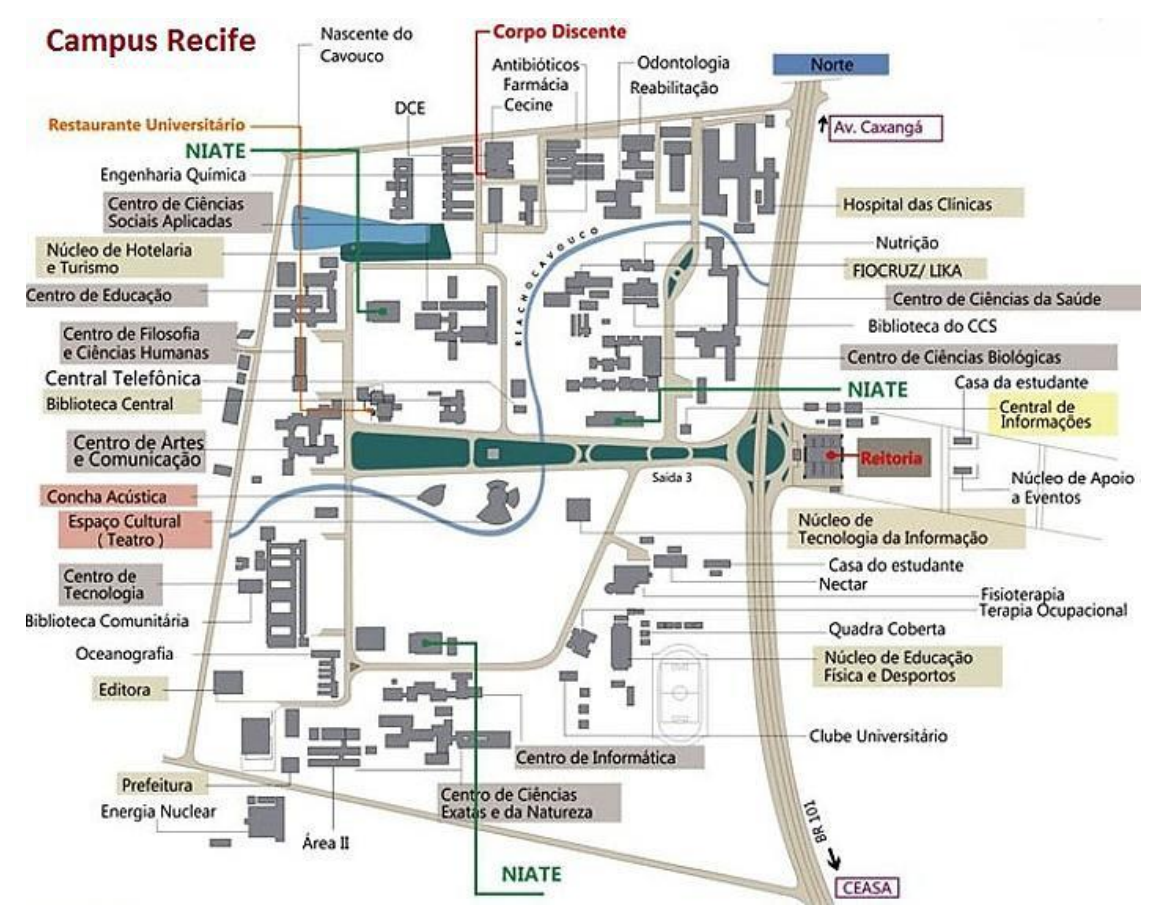

Figura 2: Área de estudo, campus da UFPE em Recife (UFPE, 2016).

\section{Dados e ferramentas}

O ponto de partida da aplicação contou com a utilização dos seguintes materiais e recursos computacionais, a saber:

- 08 (oito) Plantas topográficas cadastrais da Unificação das Bases Cadastrais (UNIBASE), restituídas em escala 1/1000, anos 1989 a 1998, SAD69 (South American Datum 1969), projeção UTM (Universal Transversa de Mercator);

- 08 (oito) Ortofotocartas da cobertura aerofotogramétrica contratada pela Prefeitura da Cidade do Recife, restituídas em escala 1/1000, ano 2007, SIRGAS2000 (Sistema de Referência Geocêntrico para as Américas), projeção UTM (Universal Transversa de Mercator);

- Nuvem de pontos do perfilamento a laser aerotransportado (LiDAR - Light Detection and Ranging), contratado pela Prefeitura da Cidade do Recife, densidade média de 5,51 pontos por $\mathrm{m}^{2}$, precisão vertical nominal melhor ou igual a $11 \mathrm{~cm}$, ano 2013, Sistema Geodésico Brasileiro: SIRGAS2000 (Sistema de Referência Geocêntrico para as Américas) e Referencial Altimétrico vinculado ao marégrafo de Imbituba-SC;

- Modelo Digital do Terreno (MDT);

- Esri ArcGIS Desktop 10; Bentley Systems MicroStation V8i (SELECTseries 3) Version 08.11.09.578; Trimble SketchUp Pro 2015 Version 15.0.9350; Bienetec S. L. Aumentaty Author Version 1.3. 


\subsection{Integração das Bases Cartográficas}

O primeiro passo da pesquisa experimental foi a compatibilização das bases cartográficas, uma vez que os documentos cartográficos se encontravam em referenciais geodésicos distintos. Os parâmetros de translação e a formulação matemática envolvidos na transformação de sistemas de coordenadas geodésicas atentaram para o que se estabelece nas especificações do Instituto Brasileiro de Geografia e Estatística (IBGE, 2016).

A partir das rotinas de projeção e transformação do ArcGIS Desktop 10 realizou-se a transformação das Plantas Topográficas Cadastrais de Referencial SAD69 para o atual Sistema Geodésico Brasileiro SIRGAS2000, projeção UTM. A transformação resultou em pequena discrepância posicional nos elementos vetoriais quando se comparam as bases cartográficas (UNIBASE e Ortofotocarta). A discrepância pode estar associada às redes geodésicas que não são homogêneas em todo o territorial nacional, à acuidade visual no processo de restituição fotogramétrica, ou à etapa de ortorretificação. Em seguida foi realizada a adequação da articulação das folhas Topográficas Cadastrais do Projeto UNIBASE (Roberto, 2003). Como o projeto foi executado em anos distintos, verificou-se uma descontinuidade nos elementos vetoriais em algumas folhas adjacentes em decorrência de omissões ocasionadas por copas de árvores e sombras na etapa de restituição.

A correção topológica foi realizada por meio do MicroStation V8i no fechamento de polígonos e linhas partidas. $\mathrm{O}$ ajustamento vetorial de polígonos resultou no fechamento das figuras, isto é, tornando idêntico o vértice de início e fim, garantindo que as coordenadas iniciais e finais sejam numericamente idênticas, definindo entidades topologicamente fechadas (regiões). A integração das bases cartográficas consistiu-se da junção de todas as folhas do projeto UNIBASE e Ortofotocartas num arquivo único. A preparação acarretou a correção de sobreposição vetorial, a alteração nas incoerências de atributos (espessura, cor, estilo e camada) e a eliminação de informações de níveis desnecessários à proposta da pesquisa (reticulado, declinação magnética, convergência meridiana e outras informações).

$\mathrm{O}$ arquivo único da base cartográfica unificada permitiu a junção vetorial de elementos idênticos em polígonos fechados (quadra, lote, lagoa, arruamento, área verde, entre outras feições de geometria fechada), e com base nele, desenvolveu-se a construção e o agrupamento de níveis de informações planimétricos, tão somente, referentes às classes de edificação, vegetação, hidrologia, via pavimentada, calçada e área não pavimentada, Figura 3. 


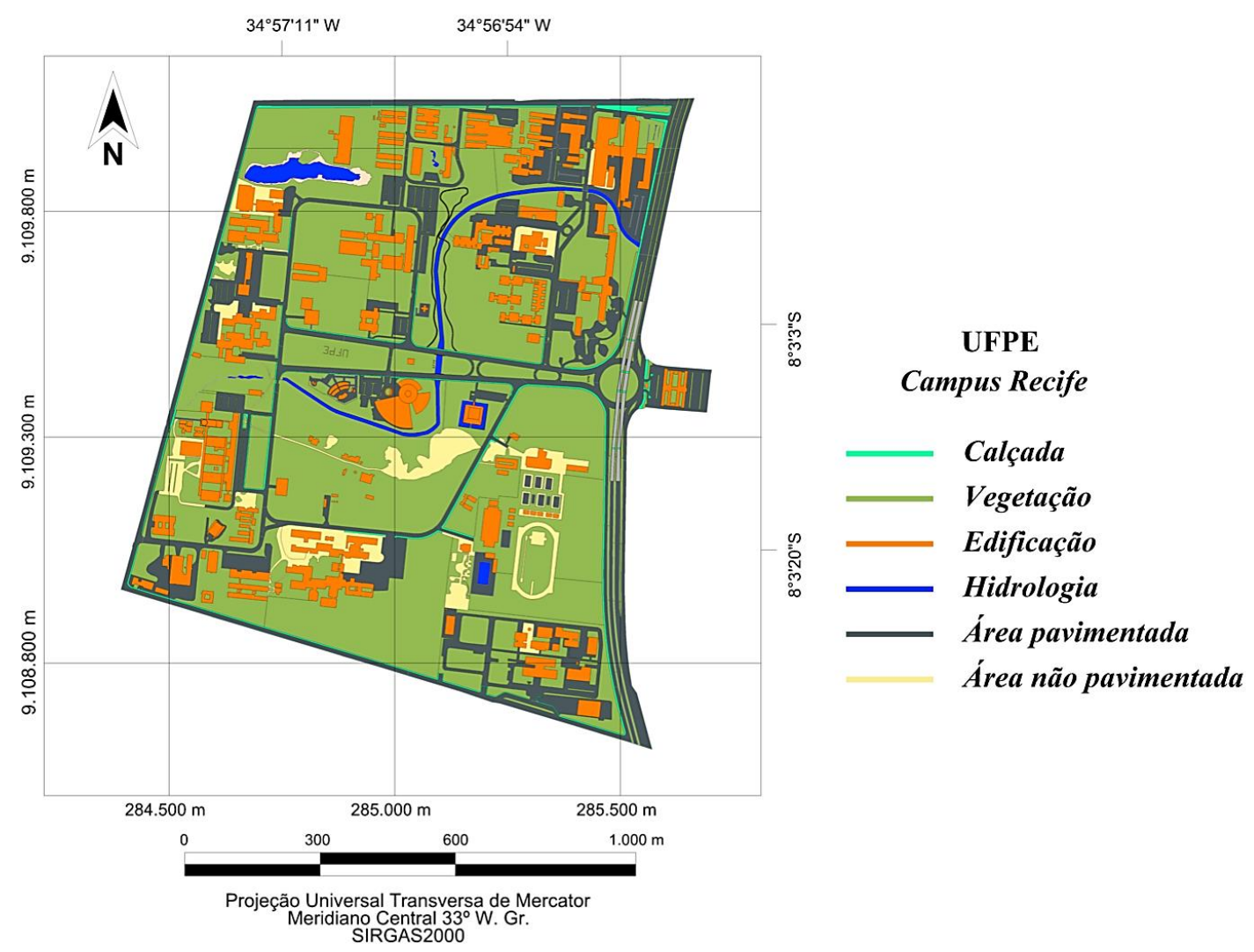

Figura 3: Base cartográfica unificada, campus da UFPE em Recife.

\subsection{Construção do modelo 3D}

A construção do espaço acadêmico urbanizado teve início com a complementação de informações altimétricas à base cartográfica unificada, isto é, com a junção de entidades vetoriais pontuais (nuvem de pontos) e Modelo Digital do Terreno à base de dados espaciais composta de objetos fechados (polígonos). A compatibilização entre os produtos foi conduzida por meio de sobreposição de informações, 2D e 3D, planialtimétricas georreferenciadas. Nesta fase, optou-se por trabalhar por bloco de malha de pontos (dimensões $100 \mathrm{~m}$ x $100 \mathrm{~m}$ ) em virtude da grande quantidade de pontos e o correspondente tempo de processamento dos dados.

A modelagem tridimensional foi implementada com o auxílio do MicroStation V8i. Por meio de filtragem eliminaram-se os pontos em topo de árvore e em linha de transmissão, possibilitando que a identificação da altura das edificações fosse referenciada ao Modelo Digital do Terreno. A partir de comandos de construção de objetos sólidos foi possível transformar as entidades 2D da base cartográfica unificada em elementos 3D, ou seja: elevações de prédios, de calçadas e de viaduto, tornando essas representações mais próximas dos objetos do mundo real. A modelagem 3D deste artigo não visa a investigação de detalhes arquitetônicos das edificações (fachadas, esquadrias, paredes e outros), ou inserções de vegetação (árvores, gramados), de placas de sinalização e de postes de energia, mas sim o espaço construído retratado no ambiente do modelo, acrescido por tonalidades de cores e texturas, Figura 4. 


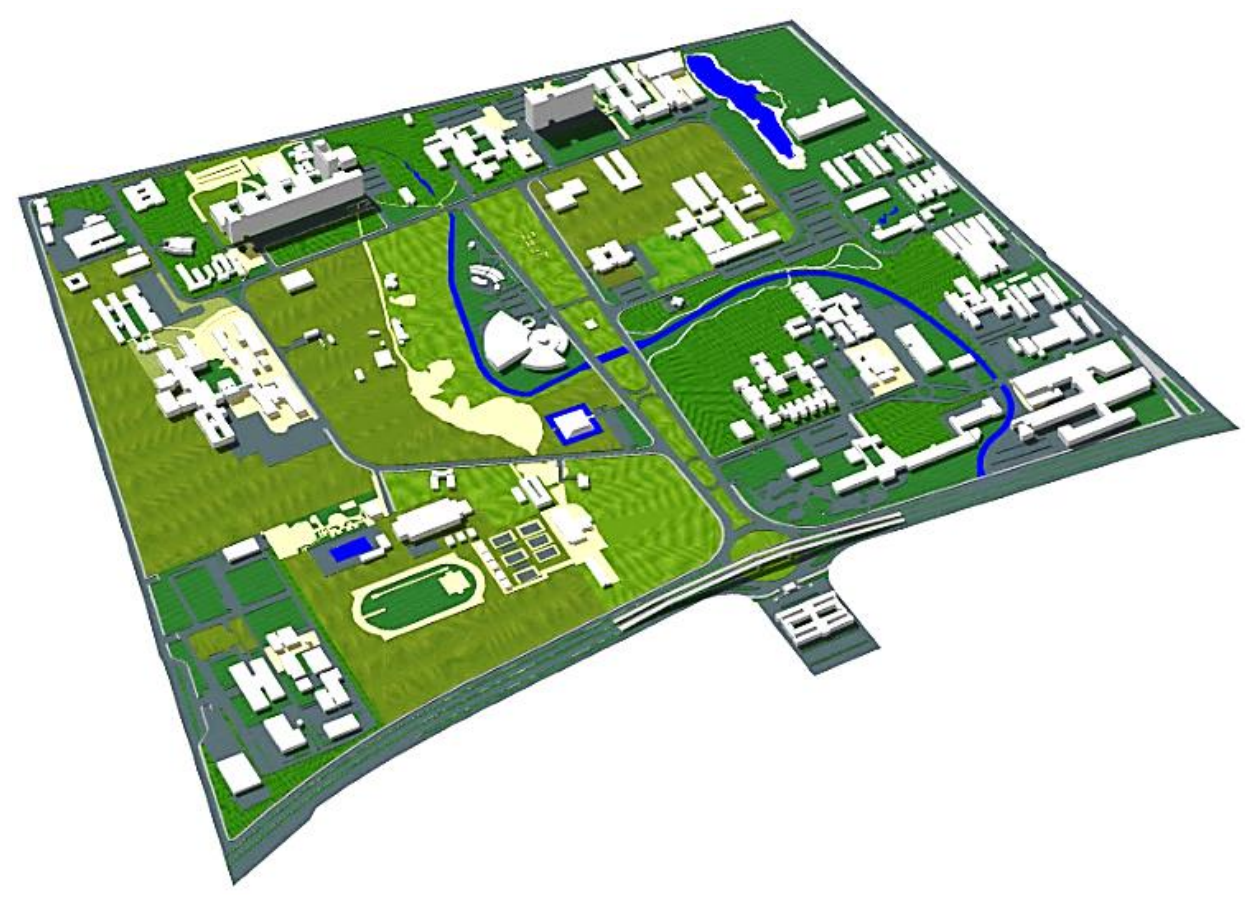

Figura 4: Vista perspectiva do Modelo 3D, campus da UFPE em Recife.

\subsection{Desenvolvimento em Realidade Aumentada}

Uma vez criado o modelo 3D em formato (.dgn), o mesmo foi exportado como arquivo .skp para leitura no Sketchup Pro. As informações originais e as adicionadas ao modelo durante seu desenvolvimento se conservaram no processo de transferência, ou seja, em termos de posicionamento, orientação, escala, cor e textura. A inserção por símbolos 3D (árvores, postes de energia, placa de sinalização, parada de ônibus, entre outros) ao ambiente construído (modelo 3D) foi omitida em função dos propósitos da pesquisa. A complexidade de cenas exige maior tempo de renderização em função da quantidade de informações gráficas referentes aos elementos.

Em seguida, por meio do Sketchup Pro, fez-se a exportação do modelo 3D em extensão (.3ds). A escolha por este formato deu-se em funções da preservação dos elementos construídos na modelagem e de ser um arquivo de tamanho reduzido. O uso em Realidade Aumentada foi desenvolvido a partir da versão gratuita do Aumentaty Author, da Bienetec S. L., o que possibilitou a detecção e rastreamento de algo real com elementos virtuais por sobreposição. Isso permitiu o relacionamento do marcador previamente cadastrado ao modelo 3D.

O programa Aumentaty Author apresenta interface gráfica intuitiva e dispensa desenvolvimento em linguagem de programação, facilitando o uso por usuários menos experientes. A versão não comercial do aplicativo apresenta opções interativas, como por exemplo: rotações (frontal, vertical e lateral), translações (aproximação e afastamento) e escala (proporção), o que permite maior dinamismo na visualização do experimento. Ressalta-se que o tamanho e o posicionamento do marcador não exerceram influência no resultado final da visualização. Foi possível nos ensaios realizar ajustes de movimentação, de deslocamento e de tamanho do modelo 3D com o marcador estático. 


\section{Resultados e aplicações da pesquisa}

A Figura 5 apresenta o resultado em Realidade Aumentada em perspectivas do campus da UFPE em Recife. A identificação e o rastreamento do marcador impresso, apresentado em tela, se sobrepõe a reprodução do modelo 3D (construído a partir de dados e produtos cartográficos). A análise visual contextualizada pode ampliar o aspecto cognitivo do observador, facilitando a percepção e o entendimento das informações retratadas, Figura 6.

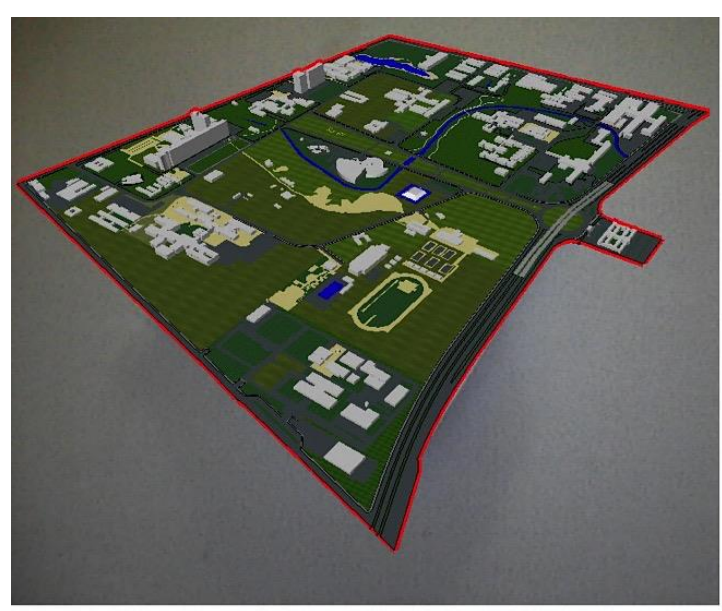

(a)

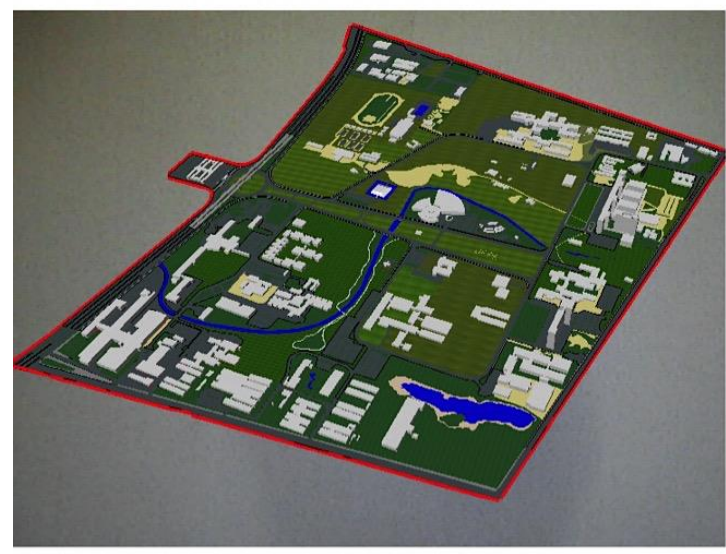

(c)

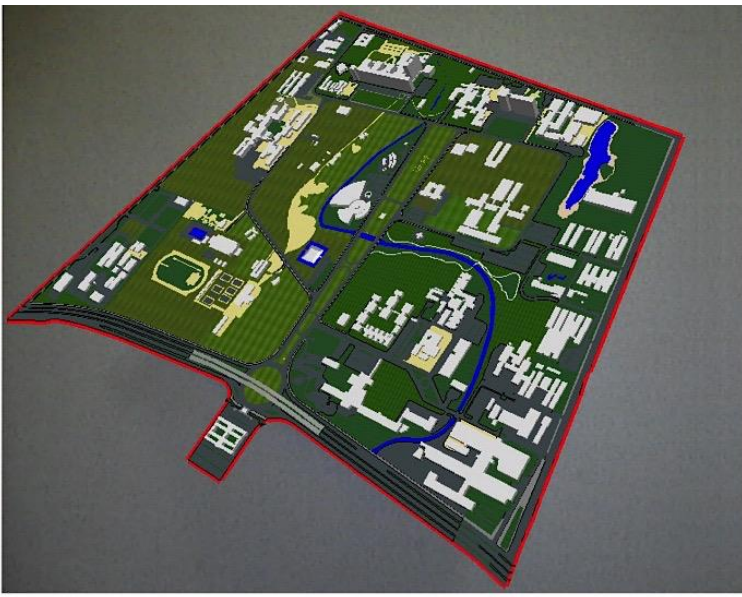

(b)

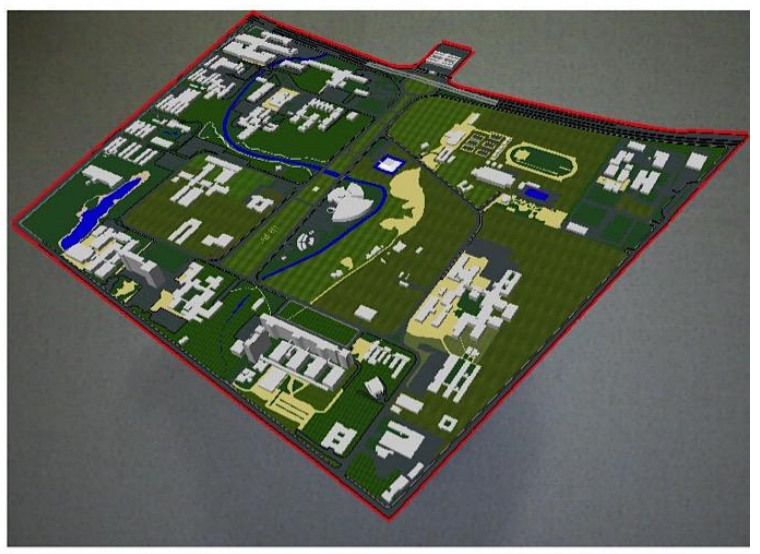

(d)

Figura 5: Resultado da Realidade Aumentada (modelos 3D) em vistas perspectivas (a), (b), (c) e (d); campus da UFPE em Recife. 


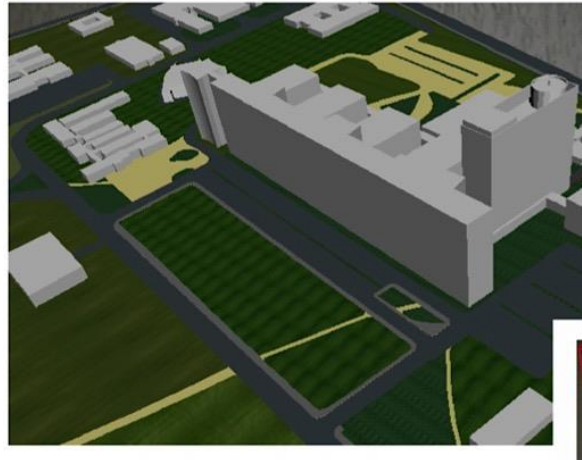

(a)

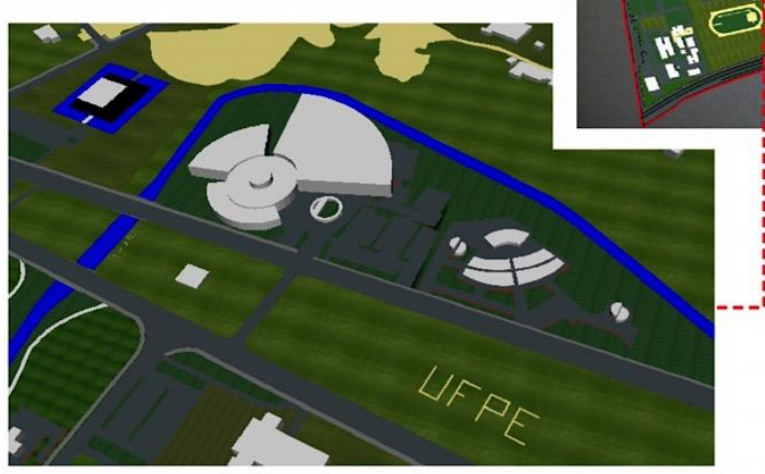

(c)

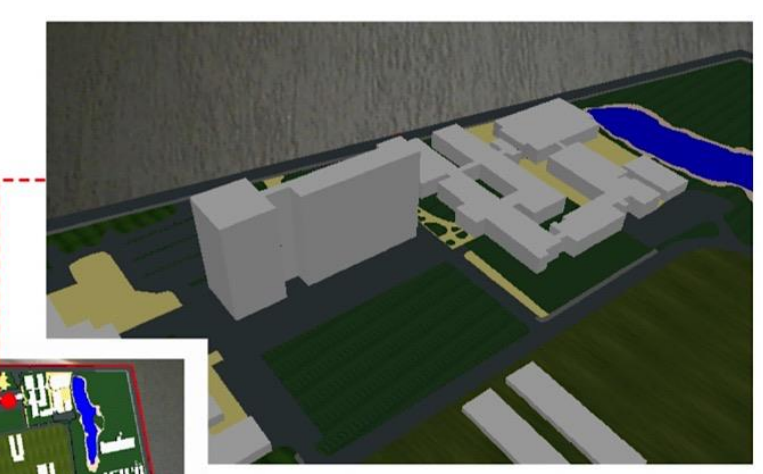

(b)
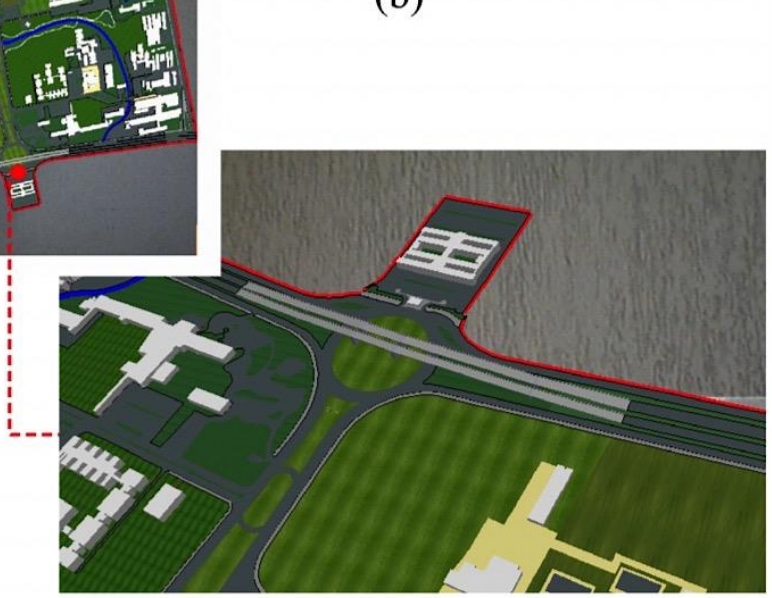

(d)

Figura 6: Identificação de edifícios no campus da UFPE com auxílio da RA. (a) Centro de Tecnologia e Geociências, (b) Centro de Filosofia e Ciências Humanas, (c) Centro de Convenções, e (d) Reitoria Universitária.

\subsection{Experimento realizado}

O experimento realizado buscou analisar como os alunos do Curso de Engenharia Cartográfica e de Agrimensura da Universidade Federal do Piauí (UFPI) responderam a um ambiente educacional de Realidade Aumentada. Para a realização deste experimento, foram selecionados 32 alunos ingressantes no período letivo 2016.1, matriculados na disciplina de Introdução ao Curso de Engenharia Cartográfica e de Agrimensura, e considerados para este fim um grupo homogêneo de usuários não especialistas.

Dos alunos selecionados, $78 \%$ têm idade ente 18 e 25 anos, $16 \%$ têm menos de 18 anos, e $6 \%$ mais de 25 anos. No grupo, 50\% cursaram o Ensino Médio em escola pública, e $50 \%$ em escola particular. Quanto a data de conclusão do Ensino Médio, $41 \%$ concluíram há menos de 01 ano, $6 \%$ há mais de 05 anos e o restante, $53 \%$ dos alunos, concluíram o Ensino Médio na faixa de 01 a 05 anos.

Dos entrevistados, 59\% informaram que na sua formação acadêmica tiveram contato moderado com noções elementares de cartografia (tonalidade, textura, forma, orientação, localização, projeção, escala, representação bidimensional e tridimensional, título, legenda, leitura de mapas, 
etc.). Outros $34 \%$ informaram contato pouco frequente, e $6 \%$ informaram contato frequente. Quanto à frequência de uso de mapas e/ou de sistemas de navegação, 53\% dos alunos informaram que usam moderadamente mapas e/ou sistemas de navegação, $38 \%$ informaram que usam com pouca frequência, e $9 \%$ informaram que usam frequentemente.

Ressalva-se que o experimento com dados do campus da UFPE foi aplicado tão somente a alunos da UFPI, visto que a prática objetivou explorar a percepção e o entendimento do ambiente real estudado, considerando-se ainda que tais alunos não deveriam possuir conhecimento prévio da área de estudo. Assim, em um primeiro momento, foi apresentada aos alunos a planta topográfica cadastral da cidade universitária, Figura 7, com as convenções cartográficas de identificação de elementos. Em seguida, os mesmos alunos manipularam a RA do campus da UFPE, conforme exemplo das Figuras 5 e 6, para confrontação e análise das informações. Por fim, os alunos responderam a um questionário comparativo. O questionário buscou explorar os seguintes aspectos qualitativos: aspecto territorial, interpretação de elementos, e aspecto comunicativo dos materiais apresentados.

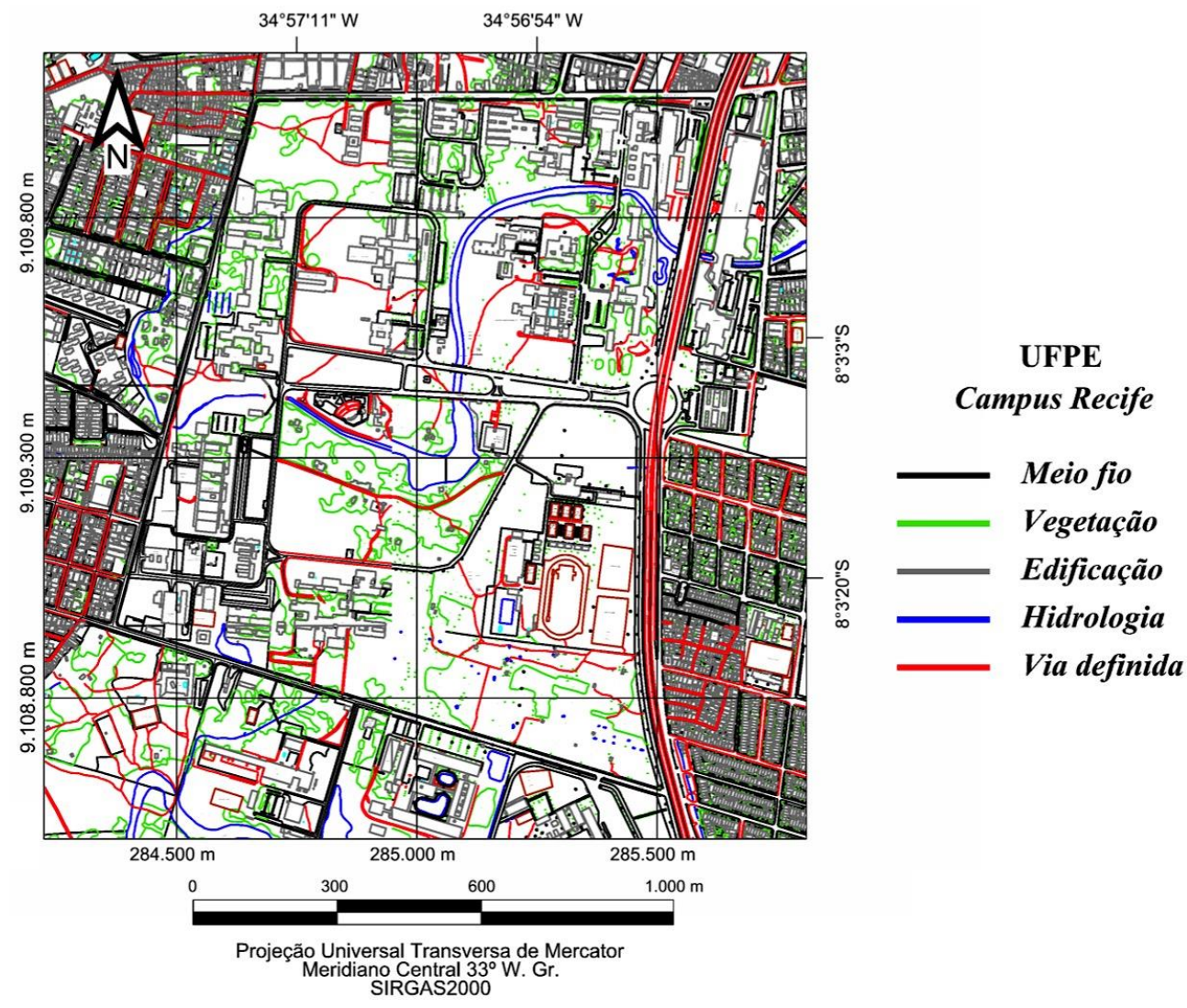

Figura 7: Planta topográfica cadastral, campus da UFPE em Recife.

$\mathrm{Na}$ percepção territorial, as respostas do questionário mostram que $59 \%$ dos entrevistados perceberam no modelo RA uma melhora significativa no aspecto territorial da área urbanizada ou dos espaços de lazer, enquanto $41 \%$ deles consideraram neste quesito que o modelo RA é igual ou pior que a planta topográfica cadastral da região. Na interpretação de elementos, 72\% dos alunos conseguiram melhor identificar no modelo RA elementos como Meio fio, Vegetação, Edificação, Hidrografia e Via definida, e outros $28 \%$ informaram que este processo de identificação é igual ou pior quando comparado à planta topográfica cadastral. 
Aproximadamente $75 \%$ dos alunos perceberam uma melhora no aspecto comunicativo do modelo RA, quando considerados aspectos como Interação, Dinamismo, Textura, Cor, e Forma e tamanho. Os demais $25 \%$ consideraram que na comparação do modelo RA com a planta topográfica cadastral tais aspectos comunicativos são iguais ou piores. De forma geral, percebeuse ainda grande interesse e curiosidade dos alunos ao manipular o modelo RA, e durante todo o processo de apresentação do material referente à Realidade Aumentada surgiram vários questionamentos, colocações e discussões a cerca da informação e da tecnologia.

\section{Conclusões}

A Realidade Aumentada tem se tornado uma interessante oportunidade para a cartografia. Apesar das diferenças conceituais entre o mapa e sua apresentação em RA, existem também uma série de similaridades a serem exploradas e ajustadas. No âmbito educacional, da cartografia, a RA mostrou-se apta a estimular o aprendizado e despertar a curiosidade do aluno em relação ao conteúdo lecionado. Diante disso, o uso da realidade aumenta pode ser eficaz na visualização de dados que exijam a transmissão dos conhecimentos representados em mapas, cartas e plantas topográficas, tendendo a beneficiar o contato do usuário com o ambiente do mundo real aumentado.

Os resultados deste artigo evidenciam a viabilidade de utilização da Realidade Aumentada na área da cartografia. O modelo RA supre a carência da visão espacial por parte de usuários não especialistas. A tecnologia pode auxiliar o ensino de relações topológicas e de camadas de informações em mapas, cartas e plantas topográficas. Percebe-se que dos aspectos analisados no experimento, os comunicativos (Interação, Dinamismo, Textura, Cor, e Forma e tamanho) são os que melhor se beneficiam do uso da RA. No entanto, experimentos desta natureza devem ser ampliados, com aplicação em diferentes conjuntos de usuários e em diferentes contextos.

Assim, emergem possibilidades de atuação da Realidade Aumentada no desenvolvimento de aplicações que requeiram um maior nível de detalhamento e interação. Com isso, criam-se oportunidades de apresentação de uma base cartográfica aumentada, como por exemplo, do relevo, evidenciando-se as formas e feições na visualização de curvas de nível, modelo digital do terreno e de superfície, e de relações topológicas, com margem para uma melhor visualização de toponímias (morro, serra, talvegue, vale e outros). Outro exemplo é o uso da RA na interpretação de elementos hidrológicos do tipo lagoa, mangue, bacia, rios, e no entendimento de afluentes, nascentes, jusante, foz, na visualização do fluxo d'água, no estudo de enchentes, nível d'água em bacias e reservatórios, e em outras abordagens.

\section{AGRADECIMENTOS}

Os autores agradecem a Agência CONDEPE/FIDEM e a Prefeitura da Cidade do Recife pela disponibilização dos dados cartográficos originais utilizados no artigo. 


\section{REFERÊNCIAS BIBLIOGRÁFICAS}

Azuma, R. T. "A survey of augmented reality." Presence 6, 4 (1997): 355-385.

Bailey, J. E.; Chen, A. "The role of virtual globes in geoscience." Computers \& Geosciences 37 , 1 (2011): 1-2.

Basogain, X.; Olabe, M.; Espinosa, K.; Rouèche, C.; Olabe, J. C. "Realidad Aumentada en la Educación: una tecnología emergente." 7a Conferencia Internacional de la Educacion y la Formacion basada en las Tecnologias, Madri, 2007.

Bodenhamer, D. J. "Beyond GIS: The Promise of Spatial Humanities." Indianápolis: Purdue GIS Day, 2014.

Cadavieco, J. F.; Goulão, M. F.; Costales, A. F. "Using augmented reality and m-learning to optimize students performance in higher education." Procedia-Social and Behavioral Sciences 46 (2012): 2970-2977.

Cardoso, A.; Junior, E. L.; Kirner, C.; Kelner, J. "Conceitos de Realidade Virtual e Aumentada." Tecnologias para o Desenvolvimento de Sistemas de Realidade Virtual e Aumentada. Cap 1 (2007): 1-16.

Carvalho, P. R. C. "Estudo das distorções do sistema geodésico de referência da Região Metropolitana do Recife." Universidade Federal de Pernambuco, 2003.

Cruz-Neira, C.; Sandin, D. J.; DeFanti, T. A. "Surround-screen projection-based virtual reality: the design and implementation of the CAVE." Proceedings of the 20th annual conference on Computer graphics and interactive techniques. Anaheim, 1993.

Delgado, A. L. N.; Olguín, C. J. M.; Ricarte, I. L. M. "Monitoring learners activities in a collaborative environment." Proceedings. Seventh International Workshop on Groupware. Washington, 2001.

DiBiase, D.; MacEachren, A. M.; Krygier, J. B.; Reeves, C. "Animation and the role of map design in scientific visualization." Cartography and geographic information systems 19, 4 (1992): 201-214.

Fosse, J. M. "Representação Cartográfica Interativa Tridimensional: Estudo da variável visual cor em ambiente VRML." diss., Universidade Federal do Paraná, 2004.

Green, S. A.; Billinghurst, M.; Chen, X.; Chase, J. G. "Human-robot collaboration: A literature review and augmented reality approach in design." International Journal of Advanced Robotic Systems, 2008.

Harwood, A. R.; Lovett, A. A.; Turner, J. A. "Customising virtual globe tours to enhance community awareness of local landscape benefits." Landscape and Urban Planning 142 (2015): 106-119.

Hearnshaw, H. M.; Unwin. D. J. Visualization in geographical information systems. New York: John Wiley \& Sons Ltd, 1994.

Henderson, S. J.; Feiner, S. K. Augmented reality for maintenance and repair (armar). DTIC Document. Technical Report AFRL-RH-WP-TR-2007-0112, United States Air Force Research Lab, 2007.

Henderson, S. J.; Feiner, S. K. "Exploring the benefits of augmented reality documentation for maintenance and repair." Visualization and Computer Graphics, IEEE Transactions on 17, 10 (2011): 1355-1368. 
IBGE. Instituto Brasileiro de Geografia e Estatística. "Sistemas de Coordenadas Geodésicas." In Geociências. Rio de Janeiro, 2016. http://www.ibge.gov.br/home/.

Kirner, C.; Kirner, T. G.; Júnior, N. C.; Buk, C. V. "Uso de realidade aumentada em ambientes virtuais de visualização de dados." Proc. of VII Symposium on Virtual Reality, São Paulo, 2004.

MacEachren, A. M. How Maps Work: Representation, Visualization, and Design. New York: Guilford Press, 2004.

MacEachren, A. M.; Kraak, M. "Exploratory cartographic visualization: advancing the agenda." Computers \& Geosciences 23, 4 (1997): 335-343.

MacEachren, A. M.; Taylor, D. R. F. Visualization in modern cartography. New York: Elsevier, 2013.

Meneguette, A. A. C. "Cartografia no século 21: revisitando conceitos e definições." Geografia e Pesquisa 6, 1 (2012): 6-32.

Miyake, R. K.; Zeman, H. D.; Duarte, F. H.; Kikuchi, R.; Ramacciotti, E.; Lovhoiden, G.; Vrancken, C. "Vein imaging: a new method of near infrared imaging, where a processed image is projected onto the skin for the enhancement of vein treatment." Dermatologic surgery 32, 8 (2006): 1031-1038.

Pinto, F. S.; Centeno, J. A. S. "A realidade aumentada em smartphones na exploração de informações estatísicas e cartográficas." Bol. Ciênc. Geod., sec. Artigos 18, 2 (2012): 282-301.

Portalés, C.; Lerma, J. L.; Navarro, S. "Augmented reality and photogrammetry: A synergy to visualize physical and virtual city environments." ISPRS Journal of Photogrammetry and Remote Sensing 65, 1 (2010):134-142.

Shekhar, S.; Feiner, S.; Aref, W. G. "From GPS and virtual globes to spatial computing-2020." GeoInformatica 19, 4 (2015): 799-832.

Shelton, B. E.; Hedley, N. R. "Using augmented reality for teaching earth-sun relationships to undergraduate geography students." Augmented Reality Toolkit, The First IEEE International Workshop, 2002.

Silva, L. F. F. C.; Gomes, J. V. P. "O uso de Realidade Aumentada no desenvolvimento de produtos cartográficos." Revista Militar de Ciência e Tecnologia. Rio de Janeiro, 2013.

Stanek, K.; Friedmannova, L. "Cartographically Augmented Reality." The 3rd ISDE Digital Earth Summit-Digital Earth in the Service of Society: Sharing Information, Building Knowledge-proceedings. Nessebar, Bulgaria: ISDE (2010): 1-9.

Sutherland, I. E. "A head-mounted three dimensional display." Proceedings of the December 911, 1968, fall joint computer conference, part I.

Taylor, D. R. F. "A conceptual basis for cartography: new directions for the information era." The Cartographic Journal 28, 2 (1991): 213-216.

Taylor, D. R. F. "Perspectives on visualization and modern cartography." In Visualization in modern cartography, 333-341. Oxford: Pergamon, 1994.

UFPE. Universidade Federal de Pernambuco. "Mapa da UFPE, Campus Recife." In Mapas. Recife, 2016: https://www.ufpe.br/ufpenova/images/mapa_ufpe_simples1.jpg.

Van Krevelen, D. W. F.; Poelman, R. "A survey of augmented reality technologies, applications and limitations." International Journal of Virtual Reality 9, 2 (2010):1. 
Zuffo, J. A.; Soares, L. P.; Zuffo, M. C.; Lopes, R. D. "Caverna digital-sistema de multiprojeção estereoscopico baseado em aglomerados de pcs para aplicações imersivas em realidade virtual." IV Symposium on Virtual and Augmented Reality. Florianópolis, 2001.

Recebido em novembro de 2015.

Aceito em agosto de 2016. 